\title{
Chronic lymphocytic leukaemias and non- Hodgkin's lymphomas by histological type in farming-animal breeding workers: a population case-control study based on a priori exposure matrices
}

Oriana Nanni, Dino Amadori, Claudio Lugaresi, Fabio Falcini, Emanuela Scarpi, Ariele Saragoni, Eva Buiatti

\begin{abstract}
Objectives-A population based casecontrol study was conducted in a highly agricultural area in Italy to investigate the association between chronic lymphocytic leukaemias (CLLs) and non-Hodgkin's lymphomas (NHLs), and subtypes, and exposure to pesticides in farming-animal breeding workers.
\end{abstract}

Methods-187 cases of CLLs and NHLs and 977 population controls were interviewed on medical, residential, family, and occupational history. Detailed information was collected about cultivated crops and animals bred from subjects who worked in farming and animal breeding. Information on crop diseases and pesticides used (and their quantity and duration) was also obtained. A priori jobexposure matrices were applied when a crop disease was reported, estimating the most probable pesticide and, when possible, an estimate of the cumulative dose. Odds ratios (ORs) were calculated by unconditional logistic analysis with adjustment for relevant confounders in farmers who bred animals and in farmers alone, for the main crops, types of animals, and pesticides categories. First recall and then the matrices were used for defining exposure, as it affected CLLs and NHLs and then separately on CLLs and low grade NHLs. Finally, the doseresponse was investigated for those pesticides which had shown some association. Results-No variable under study was associated with work in farming alone. In farming and animal breeding, no crop or animal showed an association with CLLs and NHLs when adjusted by exposure during childhood to farming and animal breeding (an indicator of life in a farming and animal breeding environment before the age of 13, which behaved as an independent risk variable). A non-significant association was found with stannates, arsenates, phosphates, and dichlorodiphenyl-trichloroethane (DDT) based on recall, and for stannates, arsenates, and DDT after the application of the matrices. When CLLs together with low grade NHLs were considered, the association with insecticides in general, carbamates, and phosphates became significant according to personal recall (ORs and
95\% confidence intervals (95\% CIs) $2 \cdot 46$, $1 \cdot 07-5 \cdot 63 ; 3 \cdot 08,1 \cdot 05-9 \cdot 00 ; 2 \cdot 97,1 \cdot 28-6 \cdot 91$, respectively). The application of the matrices also showed a risk of borderline significance for stannates and dithiocarbamates. A significant dose-response effect was found for phosphates (for logarithmic unit increase, OR $1 \cdot 17,95 \%$ CI 1.00-1.57); a strong trend for stannates and carbamates did not reach significance.

Conclusion-The association of CLLs and NHLs with work in farming-animal breeding is partially explained by exposure to pesticides-namely insecticides (carbamates, phosphates, and DDT) and stannates-possibly related to their use in animal breeding. The association is limited to cases of CLL and low grade NHL. The independent effect of the variable exposure during childhood suggests that early exposures, including possible contact with animals, may play a part in the pathogenic process of these neoplasms.

(Occup Environ Med 1996;53:652-657)

Keywords: exposure matrices; chronic lymphocytic leukaemias; farming-animal breeding; non-Hodgkin's lymphomas; cumulative exposure assessment

A population based case-control study of nonHodgkin's lymphomas (NHLs) and chronic lymphocytic leukaemias (CLLs) in association with farming and animal breeding has been conducted in a high risk area (Forli province, north eastern Italy). In this area, there is a high prevalence of farming and animal breeding associated with a wide use of pesticides. A priori job-exposure matrices have already been developed with the aim of estimating exposure to different chemicals; these were then integrated with the questionnaires, which used as guidelines the information on cultivated crops and on crop infestations. ${ }^{1}$ In a previous paper, it was shown that subjects working in farming associated with animal breeding are at high risk of NHLs and CLLs (OR $1.8,95 \%$ CI $1 \cdot 2-2 \cdot 6)$, particularly for CLLs and low grade NHLs (OR 2.3, 95\% CI 0.9-5.8; OR 1.7, $95 \%$ CI $1 \cdot 0-3 \cdot 1$, respectively, $v$ no association with medium and high grade NHLs). Having worked only in farming was not a risk factor, nor was work in any other occupational cate- 
gory. ${ }^{2}$ The category of farmers-animal breeders differed from the one of farmers not only because of a more likely and continuous contact with animals, but also because on average these subjects cultivated a wider variety of crops, farmed larger areas of land, had a longer median duration of employment, and personally used pesticides more often. ${ }^{2}$ The two possible explanations mentioned for the increased risk were contact with oncogenic viruses related to animal breeding and use of pesticides.

In this paper, results are presented for specific categories of pesticides, analysed first according to personal recall, and then by estimating exposure according to a priori jobexposure matrices.

\section{Materials and methods}

The study has been previously described in detail. ${ }^{2}$ Briefly, all incident cases of NHLs and CLLs in Forli province (north east Italy) between January 1987 and December 1990, excluding people positive for HIV, have been interviewed ( $\mathrm{n}=187)$, together with 977 controls selected from the general population and matched by sex and five-year age group. Compliance was $97.5 \%$ in cases and $88.0 \%$ in controls. Cases were further classified by one pathologist (AS) with a modified Kiel classification $^{3}$ in low grade $(n=56,30 \%)$, medium grade $(\mathrm{n}=34,18 \%)$, high grade $(\mathrm{n}=72$, $38 \%$ ) NHLs, CLLs ( $\mathrm{n}=23,12 \%)$, and two cases of mycosis fungoides.

The questionnaire asked for residential history, community life, dietary habits, smoking, alcohol consumption, family history of cancer, medical history, use of drugs, exposure to radiation, and work history. Exposure in childhood was recorded asking if the subject used to help the family in farming and in animal breeding before the age of 13. Results on all these sections have been reported in a previous paper. ${ }^{2}$

The work history section was organised in two parts. The first part collected information on the entire work experience and relative periods. Only occupations lasting for one year or more were recorded.

Jobs were classified under one of 21 wide working categories and then further classified according to 254 job titles (modified International Labour Office (ILO) questionnaire). Subjects who reported having been employed in farming or animal breeding for at least one year were asked to complete the second section of the questionnaire in which they were asked to provide detailed information on each of the 10 most widely cultivated crops with reference both to each farm of employment and to types of animals bred. Crops were cereals, sugar beet, grapes, apples and pears, strawberries, other fruit trees, vegetables, seeds, flowers, and garden nursery. Animals were cattle, sheeps, pigs, poultry, horses, and rabbits. Animal breeders who did not work in farming were not included in this population.

Ten forms were developed, through which questions were presented on the possible diseases or infestations for each crop, and a spe- cific list of chemicals was applied each time the subject answered positively about one of the crop diseases. For each identified pesticide, the subjects were asked about the usual dose for each treatment, the number of treatments a year, the surface area cultivated, and the period in years of cultivation. The use of pesticides and other chemicals was also asked with reference to animal breeding. The list of pesticides, including commercial names and active principles, was chosen on the basis of the frequency of use in the area and of the data available about chronic toxicity in humans. An a priori matrix had been developed for each crop and for each disease or infestation by an expert (CL). In this matrix one pesticide corresponded to one line and each of the following variables expressed as a mean value corresponded to each of the columns: weight (kg)/volume water; g/unit of surface area; number of treatments/year; and legal period of use. Finally, the last variable was the probability of use, scored by the expert for each pesticide with respect to the others mentioned in the matrix, taking into account the reported infestation and the legal period of use. The likely frequency (probability) with which each pesticide may have been used is based on data from the catalogues of pesticides producers, on the directory of widely used pesticides in farming, and on the wide experience of the phytopathologist (CL) involved in this study.

The method used to develop and apply the matrices, and results in terms of estimated information, have been presented elsewhere. ${ }^{1}$ Briefly, although almost all subjects remembered in detail the cultivated crops and the diseases or infestations, they were less often able to report the specific pesticides, or specify the amounts and the periods. The matrices were applied to the crop each time a cultivated crop and one or more disease or infestation was reported. Assuming that crop diseases were always treated and that treatment protocols were well established, only the most probable pesticide was estimated for each reported infestation. This was substituted by the next most probable pesticide if the subject explicitly excluded the use of the first one. This choice may lead to an underestimation of the number of subjects exposed to less widely used pesticides.

The category of farmers-animal breeders was represented by 278 subjects ( 61 cases and 217 controls). Agricultural workers not involved in animal breeding (250 subjects: 31 cases, 219 controls) were also considered in relation to crops cultivated and pesticides used. Furthermore, they were kept in all models as a separate category. Of the remaining 636 subjects, 148 ( 21 cases and 127 controls), although never employed as farmers-animal breeders or agricultural workers, still stated that they had been cultivating crops and used pesticides as a second job or after retirement. These subjects were excluded from the analysis. In all models, the controls were subjects never involved in farming or animal breeding (488 subjects, 74 of the 187 cases and 414 of the 977 controls interviewed). 
Table 1 Odds ratios for NHLs and CLLs in farming-animal breeding by cultivated crop and type of animal

\begin{tabular}{|c|c|c|c|c|c|c|}
\hline \multirow[b]{2}{*}{$\begin{array}{l}\text { Crop or } \\
\text { animal }\end{array}$} & \multicolumn{6}{|c|}{ Farmers-animal breeders } \\
\hline & $\begin{array}{l}\text { Cases } \\
(n=61)\end{array}$ & $\begin{array}{l}\text { Controls } \\
(n=217)\end{array}$ & $\begin{array}{l}\text { Adjusted } \\
O R^{\star}\end{array}$ & $95 \% C I$ & $\begin{array}{l}\text { Adjusted } \\
\text { ORt }\end{array}$ & $95 \% C I$ \\
\hline Cereals & 46 & 177 & 1.67 & $1 \cdot 10-2 \cdot 54$ & 0.99 & $0.58-1.69$ \\
\hline Grapes & 28 & 102 & $1 \cdot 68$ & $1 \cdot 02-2 \cdot 76$ & 1.01 & $0.55-1.83$ \\
\hline Strawberries & 15 & 43 & $2 \cdot 02$ & $1.05-3.88$ & $1 \cdot 21$ & $0.59-2.49$ \\
\hline Vegetables & 36 & 120 & $1 \cdot 75$ & $1.09-2 \cdot 78$ & 1.04 & $0.59-1.84$ \\
\hline Fruit trees & 26 & 109 & $1 \cdot 31$ & $0 \cdot 79-2 \cdot 18$ & 0.72 & $0.39-1.33$ \\
\hline Seeds & 11 & 23 & $2 \cdot 56$ & $1 \cdot 16-5.63$ & $1 \cdot 52$ & $0.65-3.75$ \\
\hline Cattle & 58 & 209 & $1 \cdot 74$ & $1 \cdot 16-2 \cdot 62$ & 1.08 & $0.62-1.88$ \\
\hline Sheep & 29 & 83 & $2 \cdot 38$ & $1.42-3.99$ & $1 \cdot 45$ & $0 \cdot 78-2 \cdot 70$ \\
\hline Pigs & 58 & 205 & $1 \cdot 77$ & $1 \cdot 18-2.66$ & $1 \cdot 11$ & $0.64-1.92$ \\
\hline Horses & 25 & 99 & $1 \cdot 58$ & $0.93-2.67$ & 0.88 & $0.47-1.66$ \\
\hline
\end{tabular}

Controls were 488 subjects who had never worked in farming or animal breeding.

*Variables in the models were: sex, age (continuous), altitude of municipality (dichotomous), familiar history of a haematopoietic cancer (dichotomous), Herpes zoster infection (dichotomous), having worked in agriculture (dichotomous).

†Variables in the models were the same as ${ }^{\star}$ and exposure during childhood.

The associations were measured by the maximum likelihood estimate of the odds ratio $(\mathrm{OR})$ as an estimate of the risk ratio (RR). Occupational variables were analysed after adjustment for sex and age, and because they were identified as significant variables in the previous analysis, altitude of municipality, first degree familial haemolymphopoietic cancer, and previous Herpes zoster infection. Casecontrol status was studied first by each type of crop and animal, then by exposure to specific pesticide categories as recalled by the subjects, finally by exposure estimated through the application of the matrices. All these variables were considered as dichotomous (yes or no). Each pesticide category was cross classified for its use in crops and in animal breeding. As in the previous analysis a significant independent risk was found for having helped in farming or animal breeding during childhood, independent of type of work in adult life (OR $2 \cdot 1,95 \%$ CI 1.45-3.0); this variable was also introduced in the models as an indicator of a possible undetermined exposure during childhood related to work in farming or animal breeding. Finally, the dose-response relation was studied for those pesticides which had shown some associations, estimating for each exposed subject a cumulative dose of exposure in $\mathrm{kg}$, as estimated through the matrices according to the following formula: chemical (g/unit of surface area) $\times$ units of surface area cultivated (n) $\times$ treatments $(\mathrm{n} / \mathrm{y}) \times$ duration of cultivation (y). In the models, the logarithmic trans- formation of the cumulative dose was used because of the skewed distribution of the exposure variables. The estimated cumulative dose was limited to a subset of subjects, as the necessary information was not available for pesticides used in animal breeding and in some questionnaires for some of the cultivated crops. Unconditional logistic regression analysis was used with case-control status or main histological type of cases as the response variable. ${ }^{4}$ The analysis was carried out with SAS.

\section{Results}

In table 1 the association between CLLs and NHLs and cultivation of each of six crops is shown, first adjusted by the usual variables and then adding to the model the variable of having helped in farming-animal breeding during childhood (exposure during childhood). In the table, the group vegetables includes sugar beet, fruit trees includes apples and pears, whereas flower cultivation and nurseries were excluded because of small numbers.

In the same table, results are presented for four types of animals (cattle, sheep, pigs, horses). All but one worker reported having bred poultry, and all but four having bred rabbits; therefore the risk for those who bred these animals was coincident with the overall risk for that work category.

Although all crops with the exception of fruit trees, and all types of animals with the exception of horses seem to be associated with a significantly increased risk of NHLs and CLLs in the first type of model, on the contrary all the effects lost significance when the variable exposure during childhood was included, although for strawberries, seeds, and sheep breeding a slightly increased non-significant OR persisted. The RR for exposure during childhood was significant in all models on crops and on animals, ranging from OR 1.80 (95\% CI $1 \cdot 14-2 \cdot 86)$ to OR $2 \cdot 20$ (95\% CI 1.43-3.39). Exposure during childhood was then systematically introduced in all the following models.

To evaluate the association between NHLs and CLLs and type of chemical used, the chemicals were grouped into six categories: arsenates, stannates, herbicides, fungicides,

Table 2 Odds ratios for NHLs and CLLs in farming-animal breeding by category of pesticide used as defined by recall and by the matrices

\begin{tabular}{|c|c|c|c|c|c|c|c|c|}
\hline \multirow[b]{2}{*}{ Pesticide } & \multicolumn{4}{|l|}{ Recall } & \multicolumn{4}{|l|}{ Matrices } \\
\hline & $\begin{array}{l}\text { Cases } \\
(n=61)\end{array}$ & $\begin{array}{l}\text { Control } \\
(n=217)\end{array}$ & $O R^{\star}$ & $95 \% C I$ & $\begin{array}{l}\text { Cases } \\
(n=61)\end{array}$ & $\begin{array}{l}\text { Control } \\
(n=217)\end{array}$ & $O R^{*}$ & $95 \% C I$ \\
\hline Arsenates & 8 & 16 & $1 \cdot 83$ & $0 \cdot 69-4 \cdot 25$ & 9 & 16 & 2.07 & $0 \cdot 80-5 \cdot 32$ \\
\hline Stannates & 5 & 6 & $2 \cdot 44$ & $0 \cdot 68-8 \cdot 76$ & 11 & 22 & 1.63 & $0.70-3.83$ \\
\hline Herbicides & 7 & 24 & 0.93 & $0 \cdot 36-2 \cdot 44$ & 13 & 46 & 0.90 & $0.42-1.92$ \\
\hline Fumigants & 33 & 116 & $1 \cdot 15$ & $0.71-1.85$ & 33 & 116 & 1.15 & $0 \cdot 71-1.85$ \\
\hline Fungicides & 21 & 70 & $1 \cdot 13$ & $0 \cdot 64-2 \cdot 00$ & 30 & 97 & 1.19 & $0.72-1.95$ \\
\hline Dithiocarbamates & 21 & 67 & 1.07 & $0.54-2.08$ & 30 & 94 & 1.13 & $0.62-2.08$ \\
\hline Insecticides & 40 & 111 & 1.43 & $0.80-2.55$ & 43 & 142 & $1 \cdot 12$ & $0.64-1.96$ \\
\hline Carbamates & 10 & 28 & $1 \cdot 26$ & $0.53-2.94$ & 11 & 29 & 1.34 & $0.58-3.06$ \\
\hline Chlorinated compounds & 30 & 84 & 1.35 & $0.74-2.49$ & 35 & 111 & $1 \cdot 15$ & $0.65-2.06$ \\
\hline DDT & 27 & 61 & 1.74 & $0.93-3 \cdot 27$ & 28 & 65 & 1.70 & $0 \cdot 91-3 \cdot 17$ \\
\hline Phosphates & 35 & 82 & $1 \cdot 70$ & $0.94-3.09$ & 42 & 121 & 1.32 & $0.75-2.73$ \\
\hline
\end{tabular}

Controls were 488 subjects who had never worked in farming or animal breeding.

* Variables in the models were: sex, age (continuous), altitude of municipality (dichotomous), familiar history of a haematopoietic cancer (dichotomous), Herpes zoster infection (dichotomous), having worked in agriculture (dichotomous), exposure during childhood (dichotomous). 
Table 3 Odds ratios for CLLs and low grade NHLs in farming-animal breeding by category of pesticide used from recall and by the matrices

\begin{tabular}{|c|c|c|c|c|c|c|c|c|}
\hline \multirow[b]{2}{*}{ Pesticide } & \multicolumn{4}{|l|}{ Recall } & \multicolumn{4}{|l|}{ Matrices } \\
\hline & $\begin{array}{l}\text { Cases* } \\
(n=31)\end{array}$ & $\begin{array}{l}\text { Control } \\
(n=217)\end{array}$ & ORt & $95 \% C I$ & $\begin{array}{l}\text { Cases * } \\
(n=31)\end{array}$ & $\begin{array}{l}\text { Control } \\
(n=217)\end{array}$ & ORt & $95 \% C I$ \\
\hline Stannates & 2 & 6 & $3 \cdot 18$ & $0.54-18.58$ & 6 & 22 & 3.06 & $0.99-9 \cdot 39$ \\
\hline Herbicides & 3 & 24 & 1.43 & $0.36-5 \cdot 69$ & 8 & 46 & $2 \cdot 10$ & $0 \cdot 77-5 \cdot 71$ \\
\hline Fumigants & 16 & 116 & $1 \cdot 17$ & $0 \cdot 61-2 \cdot 26$ & 16 & 116 & $1 \cdot 17$ & $0 \cdot 61-2 \cdot 25$ \\
\hline Fungicides & 12 & 70 & 1.42 & $0.67-3.00$ & 17 & 97 & 1.52 & $0 \cdot 79-2 \cdot 95$ \\
\hline Dithiocarbamates & 12 & 67 & $2 \cdot 13$ & $0 \cdot 85-5 \cdot 38$ & 17 & 94 & $2 \cdot 23$ & $0 \cdot 96-5 \cdot 17$ \\
\hline Insecticides & 20 & 111 & $2 \cdot 46$ & $1 \cdot 07-5 \cdot 63$ & 22 & 142 & 1.97 & $0 \cdot 88-4.39$ \\
\hline Carbamates & 7 & 28 & 3.08 & $1.05-9.00$ & 7 & 29 & 2.95 & $1 \cdot 01-8 \cdot 60$ \\
\hline Chlorinated compounds & 13 & 84 & 1.93 & $0.79-4.67$ & 16 & 111 & 1.73 & $0.75-3.98$ \\
\hline DDT & 11 & 61 & $2 \cdot 33$ & $0.93-5.85$ & 11 & 65 & $2 \cdot 16$ & $0 \cdot 86-5 \cdot 43$ \\
\hline Phosphates & 18 & 82 & $2 \cdot 97$ & $1 \cdot 28-6 \cdot 91$ & 22 & 121 & $2 \cdot 42$ & $1 \cdot 08-5 \cdot 41$ \\
\hline
\end{tabular}

*Cases with CLLs or low grade NHLs.

Controls were 488 subjects who had never worked in agriculture or animal breeding.

†Variables in the models were: sex, age (continuous), altitude of municipality (dichotomous), familiar history of a haematopoietic cancer (dichotomous), Herpes zoster infection (dichotomous), having worked in farming (dichotomous), exposure during childhood (dichotomous).

insecticides, and fumigants. Further, ditiocarbamates among fungicides were examined separately. Insecticides were also split into carbamates, phosphates and chlorinated compounds among which, dichloro-diphenyltrichloro-ethane (DDT) was examined separately because of its wide use. Table 2 shows results for each category classified by recall of the subjects and then by the application of the matrices.

The use of the matrices for estimating exposure increased the number of cases and controls classified as exposed for all categories of pesticides, with the exception of fumigants, as the fumigation process, well recalled by the subjects, was synonymous with the use of fumigants. No significant association was found with any of the pesticide categories, either from recall or the matrices.

However, a high (but non-significant) OR estimate was found before the application of the matrices for stannates (OR $2 \cdot 44,95 \% \mathrm{CI}$ $0 \cdot 68-8 \cdot 76$ ), arsenates (OR 1.83, 95\% CI 0.69-4.85), phosphates (OR $1 \cdot 70,95 \%$ CI 0.94-3.09), and DDT (OR 1.74, 95\% CI $0.93-3 \cdot 27)$. After the application of the matrices, a non-significant high risk was maintained for arsenates (OR 2.07, 95\% CI 0.80-5.32), stannates (OR $1 \cdot 63,95 \%$ CI $0 \cdot 70-3 \cdot 83$ ), and DDT (OR $1 \cdot 70,95 \%$ CI 0.91-3.17).

In table 3, the same analysis is presented for only cases of CLLs and low grade NHLs. According to the previous analysis, CLLs and low grade NHLs were identified as the histological types in which all the risks of being a farmer-animal breeder were concentrated. Models for arsenates are not presented because of small numbers.
From recall, exposure to insecticides in general, to carbamates, and to phosphates was significantly associated with CLLs and low grade NHLs (OR 2.46, 95\% CI 1.07-5.63; OR 3.08 , 95\% CI 1.05-9.00; OR 2.97, $95 \%$ CI $1 \cdot 28-6 \cdot 91$, respectively). A positive but not significant association was also suggested for dithiocarbamates (OR 2.13, 95\% CI 0.85$5 \cdot 38$ ) and for DDT (OR 2.33, 95\% CI $0 \cdot 93-5 \cdot 85)$. When the matrices were used for defining exposure, in general the point estimates of the ORs were confirmed in direction and magnitude. Stannates and dithiocarbamates carried high risks with borderline significance in these models, and carbamates and phosphates were confirmed as positively associated with the diseases. An increased non-significant $O R$ was also found for chlorinated compounds, DDT, and herbicides.

In farmers-animal breeders, the use of each category of pesticides was strongly correlated with the use of the others. Taking into account eight main categories (arsenates, stannates, herbicides, chlorinated compounds, fumigants, dithiocarbamates, carbamates, phosphates), out of 278 subjects, $21 \%$ did not use any category, only $18 \%$ used one, $24 \%$ used two to three, $25 \%$ were exposed to four to five, and $12 \%$ used more than five categories of pesticides. For this reason and because of small numbers, it was impossible to introduce in the same model more than one pesticide category to evaluate their separate effects.

The same variables analysed for those who worked in farming without having been involved in animal breeding did not show any positive association.

The cumulative dose of use (in $\mathrm{kg}$ ) was esti-

Table 4 Cumulative dose of the pesticides positively associated with CLLs and low grade NHLs by case-control status

\begin{tabular}{|c|c|c|c|c|c|c|c|c|c|c|}
\hline \multirow[b]{2}{*}{ Pesticide } & \multicolumn{4}{|c|}{ Cases* } & \multicolumn{6}{|c|}{ Controls } \\
\hline & $n$ & Mean & Median & Range & $n$ & Mean & Median & Range & ORt & $95 \% C I$ \\
\hline $\begin{array}{l}\text { Stannates } \\
\text { Dithiocarbamates } \\
\text { Carbamates } \\
\text { Chlorinated compounds } \\
\text { DDT } \\
\text { Phosphates } \\
\text { Herbicides }\end{array}$ & $\begin{array}{r}6 \\
14 \\
6 \\
12 \\
12 \\
5 \\
21 \\
8\end{array}$ & $\begin{array}{r}29 \\
1114 \\
34 \\
264 \\
237 \\
195 \\
296\end{array}$ & $\begin{array}{c}18 \\
558 \\
29 \\
64 \cdot 5 \\
115 \\
68 \\
78 \cdot 5\end{array}$ & $\begin{array}{l}<1-86 \\
27-3508 \\
6-70 \\
<1-2135 \\
15-772 \\
4-793 \\
2-971\end{array}$ & $\begin{array}{r}22 \\
88 \\
28 \\
90 \\
23 \\
102 \\
43\end{array}$ & $\begin{array}{c}23 \\
1680 \\
41 \\
78 \cdot 9 \\
138 \\
270 \\
190\end{array}$ & $\begin{array}{c}12 \\
467 \\
22 \\
16 \cdot 5 \\
46 \\
83 \\
96\end{array}$ & $\begin{array}{l}<1-108 \\
5-21600 \\
1-263 \\
<1-903 \\
2-825 \\
<1-3074 \\
1-1129\end{array}$ & $\begin{array}{l}1 \cdot 41 \\
1 \cdot 11 \\
1 \cdot 34 \\
1 \cdot 14 \\
1 \cdot 22 \\
1 \cdot 17 \\
1 \cdot 11\end{array}$ & $\begin{array}{l}0.98-2 \cdot 02 \\
0.97-1 \cdot 26 \\
0.98-1 \cdot 84 \\
0.93-1 \cdot 39 \\
0.95-1.57 \\
1.00-1 \cdot 36 \\
0.90-1 \cdot 36\end{array}$ \\
\hline
\end{tabular}

Mean, median, range in $\mathrm{kg}$, and OR for unitary increase (log scale).

Controls were 488 subjects who had never worked in farming or animal breeding.

*Cases with CLLs and low grade NHLs.

†Variables in the models were: sex, age (continuous), altitude of municipality (dichotomous), familiar history of a haematopoietic cancer (dichotomous), Herpes zoster infection (dichotomous), having worked in farming (dichotomous), exposure during childhood (dichotomous). 
mated through the matrices for stannates, carbamates, dithiocarbamates, phosphates, herbicides, chlorinated compounds, and DDT. It was possible to estimate the cumulative dose for 49 out of 53 cases and to 265 out of 307 controls. All subjects with a cumulative dose of $<1 \mathrm{~kg}$ were reported as non-exposed. Subjects with cumulative doses $\geqslant 1 \mathrm{~kg}$ were counted as exposed. The ORs and $95 \%$ CIs were calculated for the $\log$ of the increase of the cumulative dose.

Table 4 shows for each pesticide category the mean, median, and range of cumulative dose by case-control status and ORs for the logarithm of the differences between the cumulative dose of cases and that of controls.

Although only borderline significance was reached for unitary increase in the dose of phosphates (OR 1.17, 95\% CI 1.00-1.36), an association with increasing dose, based on small numbers, was also strongly suggested for stannates (OR 1.41, 95\% CI 0.97-1.26), carbamates (OR $1 \cdot 34,95 \%$ CI $0 \cdot 98-1 \cdot 84$ ), and DDT (OR 1.22, 95\% CI 0.95-1.57).

\section{Discussion}

This paper evaluates exposure to pesticides in farmers-animal breeders as a risk factor for NHLs and CLLs, compared with subjects who were never involved in farming or farming-animal breeding. In agricultural workers who were not animal breeders, no association was found with the diseases under study in analyses for the cultivation of crops and the exposure to specific pesticide categories. This confirms the results of a simplified previous analysis. ${ }^{2}$

The analysis by cultivated crop and by type of animal bred by farmers-animal breeders did not show any significant association with NHLs and CLLs when the ORs were adjusted for exposure during childhood. In fact, this variable behaved as a strong confounder, as ORs were uniformly increased for almost all crops and animals before adjustment, although the excess disappeared systematically after adjustment. The variable originates from one question: whether the subject had been involved in some farming-animal breeding activity before the 13th year of age. Exposure to unknown factor(s) during childhood is confirmed as a relevant and unexpected risk variable in the results presented here, but the nature of the exposure cannot be specified given the structure of the questionnaire.

Some studies reported an association between the use of pesticides in farmers and risk of NHLs, but some others were negative. ${ }^{6-10}$ Some studies included CLLs; in general, the high misclassification between CLLs and low grade NHLs justifies their grouping. ${ }^{1011}$ Pesticides which were found to be associated with NHLs were phenoxy herbicides, insecticides in general, chlorinated insecticides, and DDT. ${ }^{9}$ Fungicides and fumigants have also been associated with increased risks of NHLs. ${ }^{8}$ It has been noted that farmers may also be exposed to other chemicals, such as solvents, emulsifiers, fuels, and oils. ${ }^{612}$ No information on the content of solvents in pesticides was available in this study, but other working categories exposed to solvents were not at high risk. ${ }^{2}$ Among Iowa and Minnesota farmers, the increased risk of CLLs was attributed to the use of insecticides in relation with animal breeding, which could be linked with high exposure because of use in confined quarters. ${ }^{13}$ In another study, a threefold risk of NHLs in women was associated with dairy cattle, and a high risk was confirmed for women who personally handled chlorinated hydrocarbon insecticides. ${ }^{14}$ Contact with animals used for breeding has also been suspected of exposing the handler to oncogenic viruses, some of which are recognised as causal factors of animal haemolymphopoietic cancers or related diseases (Marek's disease in poultry, avian leukosis, papillomas in cattle, bovine lymphosarcomas). ${ }^{6}$ This hypothesis is reinforced by the finding that abattoir workers and possibly veterinarians and meat inspectors are at high risk of NHLs. ${ }^{1516}$

In the present study, the results for all cases by category of pesticide or by specific pesticides did not show any significant association for any of the 11 exposures considered, by either recall and matrices. The ORs for arsenates, stannates, DDT, and phosphates were increased, but not significantly. When the analysis was limited to CLLs and low grade NHLs, despite the reduction in the number of cases, a positive significant association emerged for insecticides in general, carbamates, and phosphates when recall was used, and to carbamates, phosphates, stannates, and dithiocarbamates (the last two were of borderline significance) when the matrices were applied. The risk associated with DDT was also increased, but did not reach significance. The limitation of the analysis to CLLs and low grade NHLs seems to avoid the dilution of the effect by medium and high grade NHLs.

A separate analysis referred to the excluded cases and confirmed that no association was found with any of the pesticides under study. No other study on exposure to pesticides and CLLs and NHLs reported results by this classification of histological type. In one previous study ${ }^{17}$ a higher risk was reported among those exposed to 2,4 dichlorophenoxy acetic acid $(2,4, \mathrm{D})$ for the follicular large cell variety of NHLs compared with other histological types. Although the two classifications are not immediately comparable, follicular NHLs are more often classified in the low grade category. ${ }^{18}$

The use of matrices is expected to increase the power of the study, allowing exposed classification of those farmers-animal breeders that despite their not recalling the specific use of pesticides, very likely used them. ${ }^{1920}$ It is worthwhile noting that in the present study the matrices were based both on existing knowledge of the pesticides in use in the area and on the information gathered from the questionnaire on the crop diseases and infestations, which was systematically used as a guideline for estimating the exposure to pesticide. The estimation was also based on the probability of use of the pesticide thought to be most likely 
to have been used in each infestation. Possibly, with the use of matrices most of the recall bias was avoided, as recall of cultivated crops and crop diseases is more likely to be independent of case-control status than is recall of pesticides. It is likely, however, that the problem of non-differential misclassification remained, which means that the measured risks, although quite high, are still underestimated.

This population of workers is characterised by the contemporary use of all kinds of pesticides. The high correlation among categories made it impossible to disentangle the effect of each pesticide category from the others and may explain the high number of associations found.

The hypothesis of a causal association is strengthened by the finding of a dose-response relation: this was significant only for phosphated insecticides $(11 \%$ increase of risk for each $\log$ unit of increase of the cumulative dose), but was suggested also for stannates, carbamates, chlorinated compounds, and for DDT (point estimate of the increase in risk = $41 \%, 34 \%$, and $22 \%$, respectively).

The results on pesticides favour of the hypothesis that some of the excess risk of CLLs and low grade NHLs in farmers-animal breeders is due to the wide and personal use of pesticides by this group of workers compared with other farmers. About $80 \%$ of farmers-animal breeders were estimated to have used at least one pesticide compared with $38 \%$ of farmers. Among these, phosphated insecticides were used by $41 \%$ of farmers-animal breeders and by $23 \%$ of farmers, carbamates were used by $14 \%$ of farmers-animal breeders and by $7 \%$ of farmers, DDT was used by $29 \%$ of farmers-animal breeders and by $10 \%$ of farmers. Among those who used at least one pesticide, more than $50 \%$ of the farmers-animal breeders versus $23 \%$ of the farmers reported that they usually spread it personally. The results reported here are also consistent with animal breeding itself increasing the risk of disease. The persisting effect of exposure in childhood suggests that contact with animals in childhood could have a role in the pathogenetic process, possibly due to viruses acting as oncogenic initiators or as immunosuppressors.

We thank Ms B Branzanti, Ms MG Minelli, and Ms A Giuseppone for the interviews they carried out; Ms Grainne Tierney for her critical assistance in the preparation of the text. This study was made possible by funding from the Provincia di Forli (Province of Forli), the Regione Emilia-Romagna
(Emilia-Romagna Region), the Lega Italiana per la Lotta contro i Tumori (the Italian League Against Cancer) in Milan, the Associazione Italiana per la Ricerca sul Cancro (Italian Association for Cancer Research) in Milan and the Ministero del Lavoro-Direzione Generale della Previdenza ed Assistenza Sociale (Ministry of Employment, Health, and Social Services) in Rome.

1 Nanni O, Ricci M, Lugaresi C, Amadori D, Falcini F, Buiatti E. Iterative use of a priori exposure matrices to improve the characterization of chemical exposures in agricultural work studies. Scand $\mathcal{f}$ Work Environ Health 1993;19:191-9.

2 Amadori D, Nanni O, Falcini F, Saragoni A, Tison V, Callea A, et al. Chronic lymphocytic leukaemias and nonHodgkin's lymphomas by histological type in farminganimal breeding workers: a population case-control study based on job titles. Occup Environ Med 1995;52:374-9.

3 Stanfield AG, Diebold J, Noel H, Kapanci Y, Rilke F, Kelengi G. Update Kiel classification for lymphomas. Lancet 1988; i: 292

4 Breslow NE, Day NE. Statistical methods in cancer research. Vol I. The analysis of case-control studies. Lyon: IARC, 1980. (IARC Sci Publ No 32.)

5 SAS Institute. SAS Technical report P-200, SAS/STAT software: CALIS and LOGISTIC procedures, release 6.04 Cary, NC: SAS Institute, 1990:236.

6 Pearce N, Bethwaite P. Increasing incidence of nonHodgkin's lymphoma: occupational and environmenta factors. Cancer Res 1992;52:5496s-500s.

7 Blair A, Linos A, Stewart PA, Burmeister LF, Gibson R, Everett G, et al. Comments on occupational and environmental factors in the origin of non-Hodgkin's lymphoma. Cancer Res 1992;52:5501s-2s.

8 Hoar Zahm S, Blair A. Pesticides and non-Hodgkin's lymphoma. Cancer Res 1992;52:5485s-8s.

9 Blair A, Hoar Zahm S. Cancer among farmers. Occup Med 1991;6:335-54.

10 Vineis P, D'Amore F, and the Working Group on the Epidemiology of Hematolymphopoietic Malignancies in Italy. The role of occupational exposure and immunodeficiency in B-cell malignancies. Epidemiology 1992;3 266-70.

11 Cartwright RA, Bernard SM, Bird CC, Darwin CM, O'Brien C, Richards IDG, et al. Chronic lymphocytic leukaemia: case control epidemiological study in Yorkshire. Leukemia Res 1987;12:79-82.

12 Petrelli G, Siepi G, Miligi L, Vineis P. Solvents in pesticides. Scand $\mathcal{F}$ Work Environ Health 1993;19:63-5.

13 Brown Morris LM, Blair A, Gibson R, Everett GD, Cantor KP, Schuman LM, et al. Pesticide exposures and other agricultural risk factors for leukemia among men in Iow and Minnesota. Cancer Res 1990;50:6585-91.

14 Zahm SH, Weisenburger DD, Saal RC, Vaught JB, Babbitt $\mathrm{PA}$, Blair A. The role of agricultural pesticide use in the development of non-Hodgkin's lymphoma in women. Arch Environ Health 1993;48:353-8.

15 Pearce NE, Smith AH, Reif JS. Increased risk of soft tissue sarcoma, malignant lymphoma, and acute myeloid leukemia in abattoir workers. Am $₹$ Ind Med 1988;14: 63-72.

16 Blair A, Hayes HM Jr. Mortality patterns among US veterinarians 1947-77: an expanded study. Int $\mathcal{F}$ Epidemiol narians 1947-77:

17 Weisenburger D, Hoar Zahm S, Ward M, Babbitt $P$ Holmes F, Boysen C, et al. Non Hodgkin's lymphoma associated with the agricultural use of herbicides: analysis by histologic type. 3rd Meeting of the European Association of Hematopathology. Wurzburg: 1990.

18 Longo DL, DeVita VT, Jaffe ES, Mauch P, Urba WJ. Lymphocytic lymphomas. Cancer principles and practice of oncology. 4th ed. Philadelphia: JB Lippincott, 1993: 1859-1927.

19 Siemiatycki J. Discovering occupational carcinogens in population-based case-control studies: review of findings from an exposure-based approach and a methodologic comparison of alternative data collection strategies. Recent results in cancer research. Vol 120. Berlin, Heidelberg: Springer-Verlag, 1990.

20 Siemiatycki J, Dewar R, Richardson L. Costs and statistical power associated with five methods of collecting occupation exposure information for population-based casecontrol studies. Am $\mathcal{F}$ Epidemiol 1989;130:1236-46. 